Pacific Journal of Mathematics

LINEAR TRANSFORMATIONS THAT PRESERVE THE

Peter Botha, STephen J. Pierce and William E. Watkins 


\title{
LINEAR TRANSFORMATIONS THAT PRESERVE THE NILPOTENT MATRICES
}

\author{
Peter Botta, Stephen Pierce and William Watkins
}

\begin{abstract}
Let $\mathrm{sl}_{n}$ be the algebra of $n \times n$ matrices with zero trace and entries in a field with at least $n$ elements. Let $\mathcal{N}$ be the set of nilpotent matrices. The main result in this paper is that the group of nonsingular linear transformations $L$ on $\mathrm{sl}_{n}$ such that $L(\Re)=\Re$ is generated by the inner automorphisms: $X \rightarrow S^{-1} X S$; the maps: $X \rightarrow a X$, for $a \neq 0$; and the map: $X \rightarrow X^{t}$ that sends a matrix $X$ to its transpose.
\end{abstract}

Introduction. Let $M_{n}$ be the algebra of $n \times n$ matrices over a field $K$ and let $S$ be an algebraic set in $M_{n}$. There are a number of theorems characterizing the linear maps $L$ on $M_{n}$ that preserve $S$, i.e. $L(S) \subseteq S$. For example there are results for $\{X$ : $\operatorname{det} X=0\}$ by Dieudonné [4], $\{X$ : rank $X \leq 1\}$ by Jacob [8] and Marcus and Moyls [10], the orthogonal group by Pierce and Botta [2] and other linear groups by Dixon [5]. In every instance the transformations $L$ that preserve these various algebraic sets have one of these two forms:

$$
L(X)=P X Q, \quad \text { for all } X
$$

or

$$
L(X)=P X^{t} Q, \quad \text { for all } X
$$

where $P$ and $Q$ are in $M_{n}$. There are conditions on $P$ and $Q$ which depend on the algebraic set $S$. For example if $S=\{X$ : det $X=0\}$ and $L$ is nonsingular then $P$ and $Q$ are nonsingular; if $S$ is the orthogonal group then $P Q=I$ and $P$ must be a scalar multiple of a matrix in the orthogonal group over the algebraic closure of $K$. For a good survey of further results of this type see Marcus [9].

In this paper we characterize the nonsingular linear transformations $L$ that preserve the set $\Re$ of nilpotent matrices. Since the linear span of $\Re$ is the space $\mathrm{sl}_{n}$ of matrices with trace zero, we may as well assume that $L$ is a transformation on $\mathrm{sl}_{n}$. (In order to see that $\mathscr{\varkappa}$ spans $\mathrm{sl}_{n}$, let $E_{i j}$ be the matrix whose only nonzero entry is a 1 in position $(i, j)$. The nilpotent matrices $E_{\imath j}$ and $E_{i i}+E_{i j}-E_{j i}-E_{j j}$ for $i \neq j \operatorname{span~sl}_{n}$.)

Actually we characterize all nonsingular semilinear mappings that preserve nilpotence. The main theorem can be extended to matrices with entries from an integral domain. The extension follows from a modification of a result of Chevalley [3, p. 104, Théorème 3]. 
TheOREM. Let $n \geq 3, K$ be a field with at least $n$ elements and suppose that $L$ is a nonsingular linear transformation on $\mathrm{sl}_{n}$ such that $L(\mathcal{N}) \subseteq \Re$. Then $L$ either has form (1) or (2), where $P Q$ is a non-zero scalar matrix.

Without the assumption that $L$ is nonsingular the theorem is false. Any map whose image is contained in the algebra $\mathscr{U}$ of the strictly upper triangular matrices preserves nilpotence. The proof of the theorem depends on a result of Gerstenhaber about maximal spaces of nilpotent matrices. We also use some elementary algebraic geometry and the fundamental theorem of projective geometry $[1$, p. 88 , Theorem 2.26].

LEMMA 1 (Gerstenhaber [6]). Suppose $K$ has at least $n$ elements and $\Re$ is a space of nilpotent matrices. Then $\operatorname{dim} \Re \leq n(n-1) / 2$. If $\operatorname{dim} \Re=$ $n(n-1) / 2$, then there exists a non-singular matrix $S$ such that $\mathfrak{N}=$ $S^{-1} \mathcal{Q} S$, where $\mathcal{U}$ is the algebra of strictly upper triangular matrices. Moreover, any matrix of nilindex $n$ is contained in exactly one maximal nilpotent algebra.

Tangent Spaces. Let $K[X]=K\left[X_{11}, \ldots, X_{n n}\right]$ be the ring of polynomials in $n^{2}$ variables with coefficients in $K$. For $r=1,2, \ldots, n$, let $E_{r}(X) \in K[X]$ be the $r$ th elementary symmetric function of the matrix $X=\left(X_{i j}\right)$, i.e. $E_{r}(X)$ is the sum of all principal $r \times r$ subdeterminants of $X$. We let $J$ be the ideal in $K[X]$ generated by $E_{1}(X), \ldots, E_{n}(X)$ and $\operatorname{rad} J=\left\{F \in K[X]: F^{k} \in J\right.$ for some positive integer $\left.k\right\}$. Clearly we have $\mathscr{N}=\left\{A \in M_{n}: F(A)=0\right.$ for all $\left.F \in J\right\}$. If $A \in \mathscr{N}$ then

$$
\tan (J, A)=\left\{B \in M_{n}:\left.\frac{d F}{d t}(A+t B)\right|_{t=0}=0 \quad \text { for all } F \in J\right\}
$$

and

$$
\tan (\operatorname{rad} J, A)=\left\{B \in M_{n}:\left.\frac{d F}{d t}(A+t B)\right|_{t=0}=0 \quad \text { for all } F \in \operatorname{rad} J\right\} .
$$

Both of these are vector spaces and the second is the usual tangent space at the point $A$ of the algebraic set $\Re$. Further, the second is a subspace of the first.

If $A$ and $B$ belong to $\mathscr{N}$ and are similar then their tangent spaces defined above are related by the appropriate similarity. Further note that $C \in \tan (J, A)$ if and only if $\left.(d / d t) E_{r}(A+t C)\right|_{t=0}=0$ for all $r=$ $1,2, \ldots, n$. If $A \in \mathcal{N}$ is of nilindex $n$, then, by taking $A$ into upper Jordan canonical form, one sees that the equations for $X \in \tan (J, A)$ are, up to a similarity,

$$
0=\sum_{i=0}^{n-j} X_{j+i, i+1}, \quad j=1,2, \ldots, n
$$


Therefore $\operatorname{dim} \tan (J, A)=n^{2}-n$. Since $J$ is generated by $n$ polynomials, if $N$ is of nilindex $n$ we have [7, p. 28, 37]

$$
n^{2}-n \leq \operatorname{dim} \Re \leq \operatorname{dim} \tan (\operatorname{rad} J, N) \leq \operatorname{dim} \tan (J, N)=n^{2}-n .
$$

So if $N$ is of nilindex $n$ then $\tan (\operatorname{rad} J, N)=\tan (J, N)$.

Lemma 2. If $A, B \in \mathfrak{T}$ are both of nilindex $n$ then $A B=B A$ if and only if $\tan (\operatorname{rad} J, A)=\tan (\operatorname{rad} J, B)$.

Proof. $A$ is of nilindex $n$ so its minimal and characteristic polynomials are equal. Therefore, if $A B=B A$, then $B$ is a polynomial in $A$. By the above remarks, we may assume that

$$
A=\left(\begin{array}{ccccc}
0 & 1 & 0 & \cdots & 0 \\
0 & 0 & 1 & \cdots & 0 \\
0 & 0 & 0 & \cdots & 0 \\
\vdots & \vdots & \vdots & & \vdots \\
0 & 0 & 0 & \cdots & 1 \\
0 & 0 & 0 & \cdots & 0
\end{array}\right),
$$

so

$$
B=\left(\begin{array}{ccccc}
0 & a_{1} & a_{2} & \cdots & a_{n-1} \\
0 & 0 & a_{1} & \cdots & a_{n-2} \\
0 & 0 & 0 & \cdots & a_{n-3} \\
\vdots & \vdots & \vdots & & \vdots \\
0 & 0 & 0 & \cdots & a_{1} \\
0 & 0 & 0 & \cdots & 0
\end{array}\right),
$$

where $a_{i} \in K$. Since $B$ is of nilindex $n, a_{1} \neq 0$. A direct computation shows that

$$
\left.\frac{d}{d t} E_{n}(B+t X)\right|_{t=0}=a_{1}^{n-1} X_{n 1}
$$

Hence the equation for $B$ arising from $E_{n}$ is $X_{n 1}=0$, which is the same as for $A$. One has that

$$
\left.\frac{d}{d t} E_{r}(B+t X)\right|_{t=0}=a_{1}^{r-1} \sum_{i=0}^{n-r} X_{r+i i+1}+\sum_{j=1}^{r-1} A_{j} \sum_{i=0}^{n-j} X_{j+i i+1}
$$


for suitable constants $A_{j}$ depending on $a_{1}, \ldots, a_{n-1}$. By induction, the equation for $B$ arising from $E_{r}$ is

$$
a_{1}^{r-1} \sum_{i=0}^{n-r} X_{r+i i+1}=0
$$

and since $a_{1} \neq 0$ this is the same as for $A$. Since $\tan (J, A)=\tan (\operatorname{rad} J, A)$ the results follows.

On the other hand, suppose $\tan (\operatorname{rad} J, A)=\tan (\operatorname{rad} J, B)$. We may assume $A$ is as above. Let $E_{i j}$ be the matrix with 1 in the $(i, j)$ position and zeros elsewhere. Then

$$
E_{j i} \in \tan (\operatorname{rad} J, A), \quad i>j
$$

and

$$
E_{j i}-E_{j+1 i+1} \in \tan (\operatorname{rad} J, A), \quad i \leq j .
$$

Writing $B=\left(b_{i j}\right)$, we have

$$
\begin{array}{ll}
\left.\frac{d}{d t} E_{2}\left(B+t E_{j i}\right)\right|_{t=0}=b_{i j}, \quad \text { if } i>j, & \\
\left.\frac{d}{d t} E_{2}\left(B+t\left(E_{j} i-E_{j+1 i+1}\right)\right)\right|_{t=0}= \pm\left(b_{i j}-b_{i+1 j+1}\right) & \text { if } i \leq j .
\end{array}
$$

Therefore $b_{i j}=0$ if $i>j$ and $b_{i j}=b_{i+1, j+1}$ if $i \leq j$, and $B$ is a polynomial in $A$.

LEMMA 3. If $L: \mathrm{sl}_{n} \rightarrow \mathrm{sl}_{n}$ is a nonsingular linear transformation with the property that $L(\Re)=\Re$, and $A \in \Re$, then $L(\tan (\operatorname{rad} J, A))=$ $\tan (\operatorname{rad} J, L(A))$.

Proof. The map $\tilde{L}: K[X] \rightarrow K[X]$ defined by $\tilde{L}(f)(A)=f(L(A))$ is a $K$-algebra homomorphism. Since $L$ is nonsingular and $L(\Re)=\mathscr{T}$ and $\operatorname{rad} J=\{f \in K[X]: f(N)=0$, for all $N \in \mathscr{N}\}$, we have $\tilde{L}(\operatorname{rad} J)=$ $\operatorname{rad} J$. Thus

$$
\begin{aligned}
\tan (\operatorname{rad} & J, L(A))=\left\{B \in M_{n}:\left.\frac{d f}{d t}(L(A)+t B)\right|_{t=0} \quad \text { for all } f \in \operatorname{rad} J\right\} \\
& =\left\{L(C) \in M_{n}:\left.\frac{d f}{d t}(L(A)+t L(C))\right|_{t=0} \text { for all } f \in \operatorname{rad} J\right\} \\
& =\left\{L(C) \in M_{n}:\left.\frac{d \tilde{L}(f)}{d t}(A+t C)\right|_{t=0}=0 \quad \text { for all } f \in \operatorname{rad} J\right\} \\
& =L(\tan (\operatorname{rad} J, A)) .
\end{aligned}
$$


Proof of theorem. First we observe that $L(\mathscr{T})=\mathscr{N}$. This follows from Lemma 1 of Dixon [5] and the fact that $L$ is nonsingular.

We now show that $L$ preserves nilindex $n$. If $A \in \mathfrak{T}$ and rank $A \leq n$ -2 , then $A$ kills two linearly independent vectors $v, w$. Let $\mathfrak{K}_{1}, \Re_{2}$ be maximal nilpotent algebras containing $A$ and killing $v, w$ respectively. Every maximal nilpotent algebra kills exactly one line, so $\mathfrak{K}_{1} \neq \mathfrak{N}_{2}$. By Lemma 1, $L$ maps maximal nilpotent algebras to maximal nilpotent algebras and again by lemma $1, L$ preserves the matrices of nilindex $n$.

Now we show that if $A \in \Re$ has rank one, then so does $L(A)$. Let $U$ be the unit auxiliary matrix $E_{12}+\cdots+E_{n-1, n}$.

First note that the only members of $\mathcal{Q}$ which commute with $U$ and $E_{12}$ are multiples of $E_{1 n}$. Thus the centre of any maximal nilpotent algebra is one-dimensional and is generated by a rank one matrix.

Let $A \in \Re$ have rank one. Then for some nonsingular $S, S^{-1} A S=E_{1 n}$. Let $\Re=S \mathscr{Q} S^{-1}$. Then $A$ generates the centre of $\Re$. Let $V \in \mathbb{R}$ have nilindex $n$. Then $V$ and $A+V$ have nilindex $n$ and commute. It follows from Lemmas 2 and 3 that $L(A+V)$ commutes with $L(V)$. Hence $L(A)$ commutes with $L(V)$. Since the nilindex $n$ matrices in $\Re$ generate $\Re$, $L(A)$ is in the centre of the maximal nilpotent algebra $L(\Re)$. Hence $L(A)$ has rank one.

We next define two bijections on the lines (through the origin) of $K^{n}$ and use the fundamental theorem of projective geometry. For each line $\langle v\rangle \in K^{n}$, define two $n-1$ dimensional subspaces of $\Re$ by

$$
\begin{aligned}
M(v) & =\{X \in \mathfrak{N} \mid \operatorname{Im} X=\langle v\rangle\}, \\
M^{t}(v) & =\left\{X^{t} \mid X \in M(v)\right\} .
\end{aligned}
$$

We will show that $L(M(v))=M(w)$ or $M^{t}(w)$ and $L\left(M^{t}(v)\right)=M\left(w^{\prime}\right)$ or $M^{t}\left(w^{\prime}\right)$ for some $w, w^{\prime} \in K^{n}$. The bijections will be $\varphi(v)=w$ and $\theta(v)=w^{\prime}$.

We note a few facts about $M(v)$. Any nonzero member of $M(v)$ has rank one. If $v, w \in K^{n}$, and are nonzero, then $M(v)$ and $M(w)$ are conjugate, and if $w=A v, A$ nonsingular, then $M(w)=A M(v) A^{-1}$. In tensor notation, $M(v)=v \otimes v^{\perp}$ and $M^{t}(v)=v^{\perp} \otimes v$. (Here, $\perp$ means orthogonal complement with respect to the dot product.) It is easily verified that $M(u) \cap M(v)=M(u)=M(v)$ if $u$ and $v$ are linearly dependent and 0 otherwise, and that $M(u) \cap M^{t}(v)=\langle u \otimes v\rangle$ if $u \cdot v=0$ and is 0 otherwise. Finally, observe that any $n-1$ dimensional subspace of $\Re$ with all of its nonzero matrices having rank one must be an $M(v)$ or an $M^{t}(v)$. It follows that for $v \in K^{n}$, there is a $w \in K^{n}$ such that $L(M(v))=M(w)$ or $M^{t}(w)$.

Suppose we have $v, w \in K^{n}$ with $L(M(v))=M\left(v^{\prime}\right)$ and $L(M(w))=$ $M^{t}\left(w^{\prime}\right)$. Since $n \geq 3$, pick $u$ orthogonal to $v$ and $w$. Then $M(v) \cap M^{t}(u)$ 
and $M(w) \cap M^{t}(u)$ are one dimensional. If $L\left(M^{t}(u)\right)=M\left(u^{\prime}\right)$ then $M\left(u^{\prime}\right) \cap M\left(v^{\prime}\right)=L\left(M^{t}(u) \cap M(v)\right)$ has dimension 1; which is impossible, as $M\left(u^{\prime}\right) \cap M\left(v^{\prime}\right)$ has dimension 0 or $n-1 \geq 2$. If $L\left(M^{t}(u)\right)=$ $M^{t}\left(u^{\prime}\right)$, we reach a similar contradiction. A similar argument holds when we examine the images of $M^{t}(v)$ and $M^{t}(w)$. Thus, by replacing $L$ with the map $X \rightarrow L(X)^{t}$ if necessary, we may assume that for any nonzero $v \in K^{n}, L(M(v))=M(w)$ and $L\left(M^{t}(v)\right)=M^{t}(u)$ for appropriate $u, w$ $\in K^{n}$.

Thus we define two maps $\varphi, \theta$ induced by $L$ on the lines of $K^{n}$. We have $L(M(v))=M(\varphi(v))$ and $L\left(M^{t}(v)\right)=M^{t}(\theta(v))$ for $v \in K^{n}$.

Since $L(\mathscr{\Re})=\mathscr{\Re}, L^{-1}$ also preserves nilpotence and hence $\varphi$ and $\theta$ are bijections on the lines of $K^{n}$.

Now we show that $\varphi$ and $\theta$ preserve coplanarity of lines in $K^{n}$ and thus satisfy the hypothesis of the fundamental theorem of projective geometry. Let $\left\langle u_{1}\right\rangle,\left\langle u_{2}\right\rangle,\left\langle u_{3}\right\rangle$ be three distinct coplanar lines in $K^{n}$. Then

$$
\begin{aligned}
2 n-1 & =\operatorname{dim}\left(M\left(u_{1}\right)+M\left(u_{2}\right)+M\left(u_{3}\right)\right) \\
& =\operatorname{dim} L\left(M\left(u_{1}\right)+M\left(u_{2}\right)+M\left(u_{3}\right)\right) \\
& =\operatorname{dim}\left(M\left(\varphi\left(u_{1}\right)\right)+M\left(\varphi\left(u_{2}\right)\right)+M\left(\varphi\left(u_{3}\right)\right)\right) .
\end{aligned}
$$

If $\varphi\left(u_{1}\right), \varphi\left(u_{2}\right), \varphi\left(u_{3}\right)$ are linearly independent then

$$
\operatorname{dim}\left(M\left(\varphi\left(u_{1}\right)\right)+M\left(\varphi\left(u_{2}\right)\right)+M\left(\varphi\left(u_{3}\right)\right)\right)=3 n-3
$$

and this is impossible since $n \geq 3$. Thus $\varphi\left(u_{1}\right), \varphi\left(u_{2}\right), \varphi\left(u_{3}\right)$ are coplanar and $\varphi$ satisfies the hypothesis of the fundamental theorem of projective geometry. So does $\theta$. Thus there exist semilinear maps $S$ and $T$ on $K^{n}$ such that $\varphi(u)=\langle S u\rangle$ and $\theta(u)=\langle T u\rangle$, for all nonzero $u$ in $K^{n}$.

There are linear maps $P$ and $Q$ on $K^{n}$ and automorphisms $\sigma$ and $\tau$ on $K$ such that $S v=P(\sigma v)$ and $T v=Q(\tau v)$. (The automorphisms act componentwise.) Then

$$
L(M(v))=M(P \sigma v)=P M(\sigma v) P^{-1}
$$

and

$$
L\left(M^{t}(v)\right)=M^{t}(Q \tau v)=Q^{t^{-1}} M^{t}(\tau v) Q^{t}
$$

Suppose $u \cdot v=0$. Then $\operatorname{dim}\left(M(u) \cap M^{t}(v)\right)=1$ and so

$$
\operatorname{dim}\left(M(P \sigma u) \cap M^{t}(Q \tau v)\right)=1
$$


and thus $(P \sigma u) \cdot(Q \tau v)=0$, i.e.,

$$
u \cdot \sigma^{-1}\left(P^{t} Q \tau v\right)=0
$$

Let $R$ be the semilinear map defined by

$$
R v=\sigma^{-1}\left(P^{t} Q \tau v\right)
$$

Then $u \cdot v=0$ implies $u \cdot R v=0$. Thus $R=d I$ is a scalar map, $\sigma=\tau$ and $P^{t} Q=d I$.

Replace the map $L$ with the map $X \rightarrow P^{-1} L(X) P$. Then $L(M(v))=$ $M(\sigma v)$ and $L\left(M^{t}(v)\right)=M^{t}(\sigma v)$, for all nonzero $v$ in $K^{n}$. Thus if $u \cdot v=0$ then $L(u \otimes v)=c(u \otimes v) \sigma(u \otimes v)$, where $c$ is a scalar valued function. If $v \in\left\langle u_{1}, u_{2}\right\rangle^{\perp}$, then by comparing $L\left(\left(u_{1}+u_{2}\right) \otimes v\right)$ with $L\left(u_{1} \otimes v\right)+$ $L\left(u_{2} \otimes v\right)$ we get $c\left(u_{1} \otimes v\right)=c\left(u_{2} \otimes v\right)$. Similarly if $u \in\left\langle v_{1}, v_{2}\right\rangle$, then $c\left(u \otimes v_{1}\right)=c\left(u \otimes v_{2}\right)$.

Now we show that $c$ is a constant function. Suppose that $u_{1} \cdot v_{1}=0$ and $u_{2} \cdot v_{2}=0$. Pick $v_{3} \in\left\langle u_{1}, u_{2}\right\rangle^{\perp}$. Then $c\left(u_{1} \otimes v_{1}\right)=c\left(u_{1} \otimes v_{3}\right)=$ $c\left(u_{2} \otimes v_{3}\right)=c\left(u_{2} \otimes v_{2}\right)$. Thus $c$ is a constant function say $c(u \otimes v)=k$. Then $L(u \otimes v)=k \sigma(u \otimes v)$, for all $u$, v with $u \cdot v=0$.

Since $L$ is linear, $\sigma$ is the identity automorphism. The rank one nilpotent matrices $\operatorname{span} \mathrm{sl}_{n}$ and so the theorem is proved.

REMARK. When $n=2$, the same result is obtained by a simple computation.

\section{REFERENCES}

1. E. Artin, Geometric Algebra, Interscience, New York, 1957.

2. E. P. Botta and S. Pierce, The preservers of any orthogonal group, Pacific J. Math., 70 (1977), 37-50.

3. C. Chevalley, Théorie des Groupes de Lie, Hermann, Paris, 1968.

4. J. Dieudonné, Sur une généralisation du groupe orthogonal à quatre variables, Arch. Math., 1 (1948), 282-287.

5. J. Dixon, Rigid embeddings of simple groups in the general linear group, Canad. J. Math., 29 (1977), 384-391.

6. M. Gerstenhaber, On nilalgebras and linear varieties of nilpotent matrices I, Amer. J. Math., 80 (1958), 614-622.

7. J. E. Humphreys, Linear Algebraic Groups, Springer-Verlag, New York, 1975.

8. H. G. Jacob, Coherence invariant mappings on Kronecker products, Amer. J. Math., 77 (1955), 177-189.

9. M. Marcus, Linear transformations on matrices, J. Res., Nat. Bur. of Stand., v. 75B, Nos. 3 and 4 (1971), 107-113.

10. M. Marcus and B. Moyls, Transformations on tensor product spaces, Pacific J. Math., 9 (1959), 1215-1221. 
Received March 16, 1979 and in revised form December 28, 1980. The work of the second author was partially supported by NCR Grant A-7862.

UNIVERSITY COLLEGE

TORONTO M5S 1A1 CANADA

UNIVERSITY COLLEGE

TORONTO M5S 1A1 CANADA

AND

CAlifornia State University

NORTHRIDGE, CA 91330 


\section{PACIFIC JOURNAL OF MATHEMATICS \\ EDITORS}

DONALD BABBITT (Managing Editor)

University of California

Los Angeles, CA 90024

\section{Hugo Rossi}

University of Utah

Salt Lake City, UT 84112

C. C. Moore and Arthur Ogus

University of California

Berkeley, CA 94720
J. DugundiI

Department of Mathematics

University of Southern California

Los Angeles, CA 90089-1113

R. FINN and H. SAMELSON

Stanford University

Stanford, CA 94305

ASSOCIATE EDITORS
R. ARens
E. F. BECKENBACH
B. H. NeumanN
F. WOLF
K. YoshidA (1906-1982)

\section{SUPPORTING INSTITUTIONS}

UNIVERSITY OF ARIZONA

UNIVERSITY OF BRITISH COLUMBIA

CALIFORNIA INSTITUTE OF TECHNOLOGY

UNIVERSITY OF CALIFORNIA

MONTANA STATE UNIVERSITY

UNIVERSITY OF NEVADA, RENO

NEW MEXICO STATE UNIVERSITY

OREGON STATE UNIVERSITY
UNIVERSITY OF OREGON

UNIVERSITY OF SOUTHERN CALIFORNIA

STANFORD UNIVERSITY

UNIVERSITY OF HAWAII

UNIVERSITY OF TOKYO

UNIVERSITY OF UTAH

WASHINGTON STATE UNIVERSITY

UNIVERSITY OF WASHINGTON 


\section{Pacific Journal of Mathematics}

Vol. 104, No. 1

May, 1983

Nestor Edgardo Aguilera and Eleonor Ofelia Harboure de Aguilera, On

the search for weighted norm inequalities for the Fourier transform $\ldots \ldots .1$

Jin Akiyama, Frank Harary and Phillip Arthur Ostrand, A graph and its complement with specified properties. VI. Chromatic and achromatic numbers ......................................... 15

Bing Ren Li, The perturbation theory for linear operators of discrete type . . . 29

Peter Botta, Stephen J. Pierce and William E. Watkins, Linear

transformations that preserve the nilpotent matrices .............. 39

Frederick Ronald Cohen, Ralph Cohen, Nicholas J. Kuhn and Joseph

Alvin Neisendorfer, Bundles over configuration spaces .......... 47

Luther Bush Fuller, Trees and proto-metrizable spaces . . . . . . . . . . 55

Giovanni P. Galdi and Salvatore Rionero, On the best conditions on the

gradient of pressure for uniqueness of viscous flows in the whole space . . 77

John R. Graef, Limit circle type results for sublinear equations $\ldots \ldots \ldots \ldots 85$

Andrzej Granas, Ronald Bernard Guenther and John Walter Lee,

Topological transversality. II. Applications to the Neumann problem for

$y^{\prime \prime}=f\left(t, y, y^{\prime}\right) \ldots \ldots \ldots \ldots \ldots \ldots \ldots \ldots \ldots \ldots \ldots \ldots \ldots \ldots \ldots . \ldots 5$

Richard Howard Hudson and Kenneth S. Williams, Extensions of

theorems of Cunningham-Aigner and Hasse-Evans . . . . . . . . . . . 111

John Francis Kurtzke, Jr., Centralizers of irregular elements in reductive algebraic groups

James F. Lawrence, Lopsided sets and orthant-intersection by convex

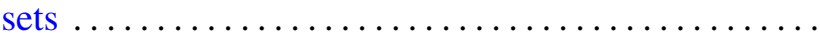

Åsvald Lima, G. H. Olsen and U. Uttersrud, Intersections of $M$-ideals and

$G$-spaces

Wallace Smith Martindale, III and C. Robert Miers, On the iterates of derivations of prime rings

Thomas H. Pate, Jr, A characterization of a Neuberger type iteration procedure that leads to solutions of classical boundary value problems

Carl L. Prather and Ken Shaw, Zeros of successive iterates of multiplier-sequence operators

Billy E. Rhoades, The fine spectra for weighted mean operators

Rudolf J. Taschner, A general version of van der Corput's difference theorem

Johannes A. Van Casteren, Operators similar to unitary or selfadjoint ones 